BMJ

Open

Gastroenterology

\title{
Tethered capsule en face optical coherence tomography for imaging Barrett's oesophagus in unsedated patients
}

\author{
Kaicheng Liang, ${ }^{1}$ Osman O Ahsen, ${ }^{1}$ Annalee Murphy, ${ }^{2}$ Jason Zhang, ${ }^{1}$ \\ Tan H Nguyen, ${ }^{1}$ Benjamin Potsaid, ${ }^{1}$ Marisa Figueiredo, ${ }^{2}$ Qin Huang, ${ }^{2,3}$ \\ Hiroshi Mashimo, ${ }^{2,4}$ James G Fujimoto (1) ${ }^{1}$
}

To cite: Liang K, Ahsen 00, Murphy A, et al. Tethered capsule en face optical coherence tomography for imaging Barrett's oesophagus in unsedated patients. BMJ Open Gastro 2020;7:e000444. doi:10.1136/ bmjgast-2020-000444

Received 15 May 2020 Revised 14 July 2020 Accepted 29 July 2020
Check for updates

(C) Author(s) (or their employer(s)) 2020. Re-use permitted under CC BY-NC. No commercial re-use. See rights and permissions. Published by BMJ.

${ }^{1}$ Department of Electrical Engineering and Computer Science, and Research Laboratory of Electronics, Massachusetts Institute of Technology, Cambridge, Massachusetts, USA

${ }^{2}$ Gastroenterology, VA Boston Healthcare System, Boston, Massachusetts, USA

${ }^{3}$ Department of Pathology, Harvard Medical School, Boston, Massachusetts, USA ${ }^{4}$ Department of Medicine, Harvard Medical School, Boston, Massachusetts, USA

Correspondence to Professor James G Fujimoto; jgfuji@mit.edu

\section{ABSTRACT}

Objective Barrett's oesophagus (BE) screening outside the endoscopy suite can identify patients for surveillance and reduce mortality. Tethered capsule optical coherence tomography (OCT) can volumetrically image oesophageal mucosa in unsedated patients and detect features of $\mathrm{BE}$. We investigated ultrahigh-speed tethered capsule swept-source OCT (SS-OCT), improved device design, developed procedural techniques and measured capsule contact, longitudinal pullback non-uniformity and patient toleration.

Design OCT was performed in 16 patients prior to endoscopic surveillance/treatment. Unsedated patients swallowed the capsule with sips of water and the tether was pulled back to image the oesophagus. SS-OCT at $1000000 \mathrm{~A}$-scans/s enabled imaging $10 \mathrm{~cm}$ oesophageal lengths in $10 \mathrm{~s}$ with $30 \mu \mathrm{m}$ transverse and $8 \mu \mathrm{m}$ axial resolution. Capsule contact, Iongitudinal image coverage and patient toleration were assessed.

Results Nine patients had non-dysplastic BE, three had ablative treatment-naïve neoplasia and four had prior ablation for dysplasia. Dry swallows facilitated capsule transit through the lower oesophageal sphincter (LES), and waiting $10 \mathrm{~s}$ before pullback reduced swallow induced LES relaxation. Slow nasal inhalation facilitated capsule retrieval and minimised gag reflex. The procedure was well tolerated. Ultrahigh-speed SS-OCT generated crosssectional and subsurface en face images showing BE features, while subsurface en face images were required to assess the gastro-oesophageal junction. Candidate features of dysplasia were also identified which could inform follow-up endoscopy/biopsy. BE features were seen in all patients with histologically confirmed BE. Mean capsule contact over BE was $75 \% \pm 27 \%$ for all patients and better in short segment $\mathrm{BE}$. Mean longitudinal image coverage over BE was $59 \% \pm 34 \%$ and better for long segment $B E$.

Conclusions Ultrahigh-speed tethered capsule SS-OCT can image en face and cross-sectional mucosal features over wide areas. Device and procedure optimisation improved performance. BE features could be identified in all patients, but limited capsule contact and longitudinal coverage could cause sampling errors for focal pathologies.
Summary box

What is already known about this subject?

- Modalities for Barrett's oesophagus screening and risk stratification outside the endoscopy suite can improve access to care and potentially reduce mortality.

- Previous optical coherence tomography (OCT) imaging studies with balloons and tethered capsules have shown diagnostic potential for BE and dysplasia but acquired sparse cross-sectional images with limited coverage and ability to visualise en face features.

- Procedural information on tethered capsule OCT has been limited.

What are the new findings?

- Ultrahigh-speed swept-source (SS)-OCT tethered capsules can generate en face and cross-sectional images, mapping wide areas of the oesophagus and gastro-oesophageal junction and enabling visualisation of $B E$ features in unsedated patients outside the endoscopy suite.

- Candidate features of dysplasia are also identified which could inform follow-up endoscopy/biopsy.

- Device design and procedural techniques are described for optimising examination performance in unsedated patients.

How might it impact on clinical practice in the foreseeable future?

- Next-generation, ultrahigh-speed SS-OCT tethered capsule imaging may improve screening for BE and risk stratification at points of care outside the endoscopy suite.

\section{INTRODUCTION}

Barrett's oesophagus (BE) and dysplasia are precursors to oesophageal adenocarcinoma (EAC). However, $90 \%$ of patients with EAC never receive an endoscopic $\mathrm{BE}$ diagnosis ${ }^{1}$ and $40 \%$ do not report symptoms of chronic gastro-oesophageal reflux disease. ${ }^{2}$ Therefore, new BE screening methods that can be used at points of care outside the endoscopy 
suite are needed to improve early detection of BE before progression to EAC. The Cytosponge has been validated for $\mathrm{BE}$ detection in large trials with $80 \%$ sensitivity and $92 \%$ specificity $^{3}$ and evaluated for risk stratification. ${ }^{4}$ Unsedated transnasal endoscopy has been compared with endoscopy, showing comparable detection of $\mathrm{BE}$, although transnasal intubation requires expertise and tolerability results have been mixed. ${ }^{56}$ Breath testing has demonstrated $\sim 80 \%$ sensitivity/specificity to BE. ${ }^{7}$ DNA methylation markers and a swallowable device for tissue sampling achieved $>90 \%$ sensitivity/specificity to BE. ${ }^{8}$

Endoscopic optical coherence tomography (OCT) can image subsurface tissue architectural morphology and early studies suggested its potential for BE and dysplasia detection. ${ }^{9}{ }^{10}$ Balloon-based OCT has been commercialised as volumetric laser endomicroscopy (VLE, NinePoint Medical). ${ }^{11-14}$ Tethered capsules can be swallowed without sedation; early string-tethered video capsules showed sensitivity and specificity of $93.5 \%$ and $78.7 \%$ compared with histological $\mathrm{BE}$ diagnosis ${ }^{15}$ and were later adapted for OCT imaging. ${ }^{16}$ Tethered capsule OCT can image long lengths of the oesophagus by slow retraction of the tether after swallowing. OCT capsule studies demonstrated cross-sectional images, reported excellent patient toleration ${ }^{17}$ and showed correlations with endoscopic Prague measurements. ${ }^{18}$ However, there are limited investigations of capsule contact with the oesophagus and longitudinal pullback non-uniformity, which reduce image coverage and cause sampling errors. Procedural details for tethered OCT capsule imaging in unsedated patients have also been limited.
Our group previously reported ultrahigh-speed OCT technology that generates en face and cross-sectional views enabling three-dimensional assessment of tissue architectural morphology ${ }^{19}{ }^{20}$ and tethered capsule OCT for imaging the oesophagus in sedated patients during endoscopy. ${ }^{21}$ Here, we report ultrahigh-speed, tethered capsule OCT for mapping the oesophagus and imaging $\mathrm{BE}$ in unsedated patients. We describe device improvements and procedural techniques for optimal imaging, measure capsule contact and longitudinal image coverage for $\mathrm{BE}$ detection and assess patient toleration.

\section{METHODS}

\section{Imaging system and tethered capsule}

Tethered capsule OCT imaging was performed using a prototype ultrahigh-speed swept-source OCT (SS-OCT) instrument operating at $1000000 \mathrm{~A}-\mathrm{scans} / \mathrm{s}, 20 \times$ faster than commercial endoscopic OCT (VLE NinePoint). The tethered capsules (figure 1A-C) used micromotors for circumferential imaging at 300 cross-sectional images per second ${ }^{21}$ with $30 \mu \mathrm{m}$ transverse and $8 \mu \mathrm{m}$ axial resolution. Several improvements were made over previously reported capsule designs. ${ }^{17}$ Our new capsule was 12 $\mathrm{mm}$ diameter with proximal and distal ends made with lubricious, medical-grade ultrahigh-molecular-weight polyethylene and a small polycarbonate transparent window for the OCT beam. This new design reduced friction, allowing the capsule to be swallowed and retrieved smoothly. The proximal end had a $30^{\circ}$ taper, improving pullback smoothness and ease of retrieval through the
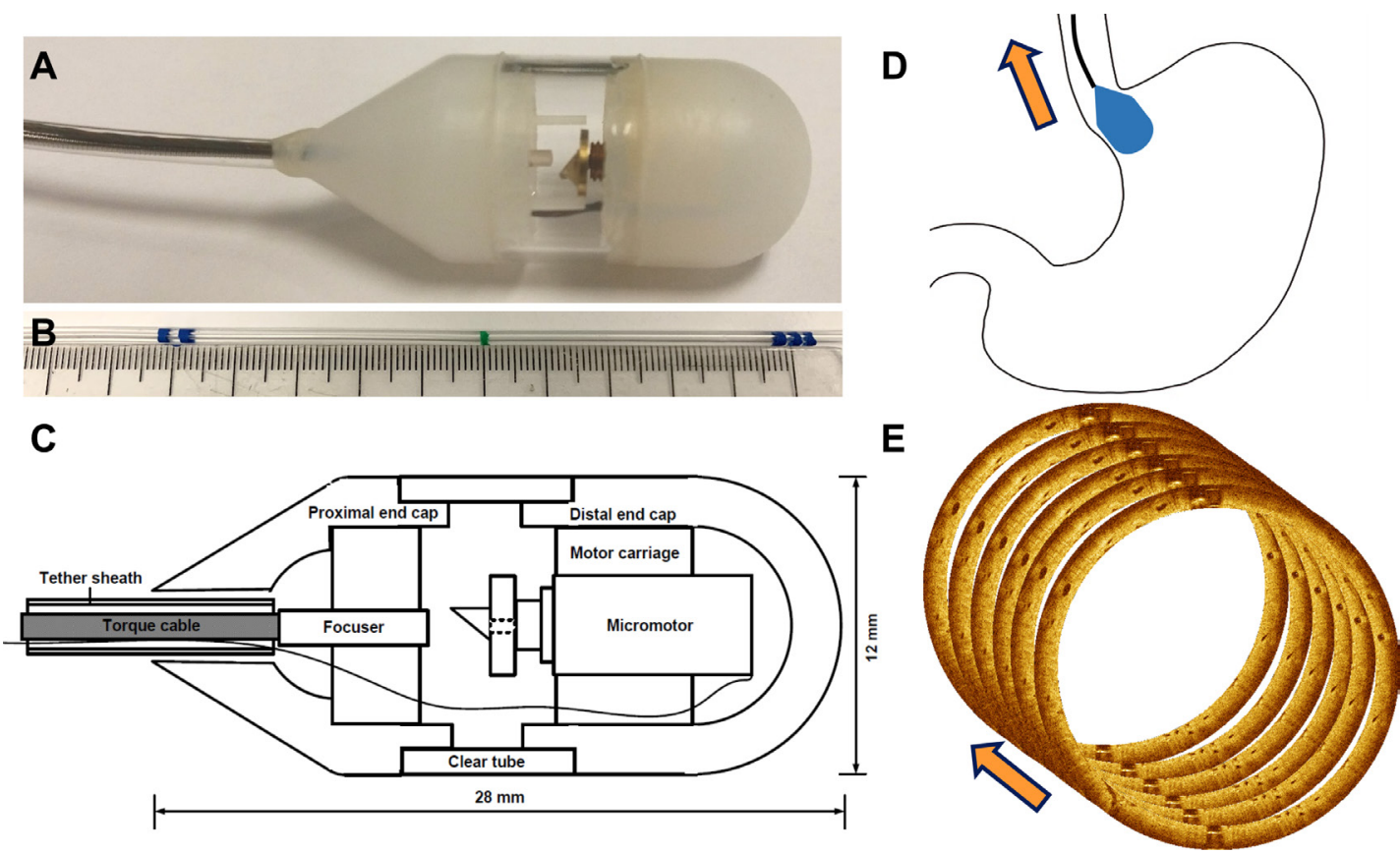

Figure 1 (A) Photograph of tethered optical coherence tomography (OCT) capsule constructed using lubricious material with a $30^{\circ}$ proximal taper for ease of retrieval. (B) Tether markings every $5 \mathrm{~cm}$ indicating distance from incisors. (C) Schematic showing micromotor rotary optical scanner and other components. (D) Cartoon showing capsule travelling from gastric cardia into distal oesophagus during a pullback image acquisition. (E) Illustration showing multiple cross-sectional images acquired in rapid succession during capsule pullback to obtain volumetric data for subsurface en face and cross-sectional visualisation. 
lower oesophageal sphincter (LES) and upper oesophageal sphincter (UES). The tether was $2.2 \mathrm{~mm}$ diameter, providing flexibility for patient comfort while retaining some rigidity for operator control, and was marked at $5 \mathrm{~cm}$ intervals to assess capsule distance from the incisors (figure 1B). The capsule device was powered by the imaging system through the tether and did not require battery power.

\section{Patient recruitment and imaging procedure}

Written informed consent was obtained from patients undergoing $\mathrm{BE}$ surveillance or endoscopic treatment for prior diagnosed neoplasia. Neoplasia was defined to include low-grade dysplasia, high-grade dysplasia and intramucosal carcinoma. Exclusion criteria were large hiatal hernia, dysphagia including suspect oropharyngeal dysphagia/aspiration based on patient history, known obstructing lesions such as strictures, masses or diverticula and inability or unwillingness to swallow the capsule. Patients were scheduled for same-day sedated endoscopy and underwent standard endoscopy preparation.

Prior to sedation and endoscopy, patients swallowed the tethered capsule with small sips of water while sitting upright. The procedure was supervised by an endoscopist who controlled the tether. Wet and dry swallows facilitated capsule transit into the stomach, and the capsule was initially positioned at the gastric cardia using real-time OCT imaging, such that the subsequent tether pullback moved the capsule through the gastro-oesophageal junction (GEJ). The endoscopist then imaged a $10 \mathrm{~cm}$ long segment of the oesophagus by pulling back the tether at $\sim 1 \mathrm{~cm} / \mathrm{s}$ for $\sim 10 \mathrm{~s}$, causing the capsule to move longitudinally through the GEJ (figure 1D). The pullback length was estimated by the tether markings. When available, previous reports were used to inform the endoscopist of the amount of $\mathrm{BE}$ present. If previous reports were not available, a long capsule pullback was performed to include both the GEJ and the squamocolumnar junction $(\mathrm{SCJ})$, thus ensuring that the entire BE segment length had been imaged. A $10 \mathrm{~cm}$ pullback fully covered the BE extent in most cases and dense volumetric datasets with $\sim 10$ million A-scans and $\sim 5$ gigavoxels, covering a $40 \mathrm{~cm}^{2}$ area of the oesophagus were acquired (figure 1E). In patients whose real-time imaging did not show the SCJ after the first pullback, a larger dataset using a longer pullback was acquired.

Patients were asked to perform dry swallows to improve capsule contact with the oesophageal wall, and the endoscopist waited $\sim 10$ s prior to the pullback to avoid swallow induced LES relaxation (deglutitive inhibition). ${ }^{22}$ Imaging was performed during pullback and not during the capsule's natural migration after swallows because peristalsis, generally progressing at several $\mathrm{cm} / \mathrm{s}$, is too rapid for dense volumetric imaging. Capsule contact with the oesophagus was assessed by observing the crosssectional image series and en face images. If substantial out of contact regions were noted, additional dry/ wet swallows were performed to improve contact before repeating pullback image acquisitions. If excessive longitudinal pullback non-uniformity occurred, as indicated by longitudinal stretching or compression of features in the en face image, additional pullback acquisitions were repeated after a brief $10-15 \mathrm{~s}$ wait period postswallow to avoid the rebound LES contractions. After imaging, the capsule was retrieved by retracting the tether. Swallowing motions were not effective for capsule retrieval because the relaxation of the UES is transient and this places the posterior tongue closer to the tether, causing gagging. Instead, patients were asked to perform slow nasal inhalation, minimising gag reflex from the posterior tongue.

Patients then underwent sedated endoscopy $\sim 2-4$ hours later, following the American Society for Gastrointestinal Endoscopy guidelines requiring 2-hour fasting after ingestion of clear liquids before intravenous sedation. ${ }^{23}$ During endoscopy, measurements were obtained of BE length and diaphragmatic hiatus. Seattle protocol 4-quadrant biopsy was performed in non-dysplastic BE (NDBE) surveillance patients, and endoscopic treatment was performed in patients with prior diagnosed dysplasia, per standard of care. In patients referred for treatment with known prior treatment-naive dysplasia, biopsies/ resections were not systematically obtained unless clinically indicated.

After tethered capsule OCT and endoscopy, a research nurse performed a brief patient interview to assess toleration for unsedated tethered capsule OCT and sedation endoscopy. The postprocedure patient interview questions were: "How anxious did you feel before the procedure? 1 not, 5 very", "How much discomfort did you have during the procedure? 1 none, 5 a lot" and "Would you recommend the procedure to others? 1 definitely yes, 5 definitely no". The questions were identical to those reported in previous OCT capsule imaging studies to facilitate comparison. ${ }^{17}$

For further clarity, the procedure can be summarised as follows:

1. Patient swallows tethered capsule with sips of water.

2. Operator monitors capsule transit through GEJ using real-time OCT imaging.

3. Patient takes dry/wet swallows to improve contact.

4. Operator waits $10-15 \mathrm{~s}$ after a swallow, then pulls back on tether (retracts capsule) to image the oesophagus from the GEJ to SCJ.

5. Repeat imaging of oesophagus if required (patient swallows, followed by steps 2-4).

6. Patient performs slow nasal inhalation and operator retrieves capsule via mouth.

7. Patient interviewed to assess procedural toleration by nurse.

8. Patient undergoes sedation and same-day endoscopy 2-4hours later if previously scheduled.

\section{Data analysis}

Datasets were assessed for image quality and one optimal dataset from each patient was selected for analysis. The region of analysis on the en face OCT images was defined 
to be the portion of the oesophageal wall that closely encircled the capsule, with proximal margin at the SCJ maximal extent and longitudinal extent as observed by endoscopy (Prague M length). The endoscopically observed $\mathrm{BE}$ length was used to help delineate the extent of BE in the en face OCT images because the GEJ is a morphological transition zone that could not be reliably delineated by OCT features alone. Since this analysis was retrospective, endoscopic measurements aided the OCT assessment. However, this suggests that quantitative measurements of $\mathrm{BE}$ length (Prague $\mathrm{M}$ length) using OCT alone will be challenging because of uncertainty in GEJ location. Analogously, endoscopic identification of the GEJ relies on discerning the top of the gastric folds, a gross anatomical landmark that is similarly prone to uncertainty. It should be noted that BE measurement would not be required in a screening procedure, because patients with features suggesting BE would be referred for follow-up endoscopy/biopsy.

The longitudinal capsule motion was non-uniform compared with the tether pullback, therefore regions where the capsule moved more slowly/rapidly could be detected as longitudinal stretching/compression of features in the en face OCT images. This longitudinal pullback non-uniformity caused distortion or gaps in the OCT data, limiting longitudinal image coverage and producing sampling errors. These artefacts also made quantitative measurements of BE length (Prague M length) challenging.

Contact of the capsule with the oesophagus and longitudinal pullback non-uniformity were analysed as metrics for image coverage and sampling error. Capsule-tissue contact was measured as the percent of the capsule circumference where the oesophagus was within $\sim 100 \mu \mathrm{m}$ from the capsule surface. This is an approximate measure of image coverage, because in regions of oesophageal folding, the extent of the folded region could not be measured. The mean of the capsule-tissue contact was calculated from the region in the en face OCT from the GEJ to the maximal extent of visible $\mathrm{BE}$ at the SCJ. Squamous mucosa regions proximal to the SCJ maximal extent were excluded from the analysis, because these areas usually showed good contact, but were less relevant to $\mathrm{BE}$ assessment.

Longitudinal image coverage was estimated by identifying longitudinally stretched regions in the en face OCT where the capsule stopped or moved too slowly relative to the oesophagus during pullback. The percentage of the image with longitudinal stretching was calculated. However, areas where the capsule moved too fast relative to the oesophagus appeared as longitudinally compressed regions in en face OCT. These regions comprise a negligible portion of the total longitudinal image but represent the sampling error. The image data are displayed as a function of time. The regions with slow capsule longitudinal motion were assumed to be approximately equal to the regions with overly fast motion because the tether pullback speed is constant and the overall pullback length and time are known. Therefore, we used the longitudinally stretched areas in the en face OCT as a marker to estimate the longitudinal image coverage and sampling error.

Tissue contact and longitudinal image coverage in the $\mathrm{BE}$ segment were reported as mean $\pm \mathrm{SD}$ over all patients, and stratified by short/long segment $\mathrm{BE}(\leq 3 \mathrm{~cm}$ and $>3 \mathrm{~cm}$ ), and absence/presence of sliding hiatal hernia (the distance of diaphragmatic hiatus from gastric folds $\leq 2 \mathrm{~cm}$ and $>2 \mathrm{~cm}$ ) subgroups.

The patient toleration (anxiety and discomfort) and recommendation scores were analysed. The toleration score distributions were stratified by pathology and treatment subgroups to show possible associations with prior endoscopy and/or treatment experience. Statistical analysis was not performed due to the small enrolment size and the observational nature of the study.

\section{RESULTS}

\section{Patient demographics and clinical characteristics}

Sixteen patients were enrolled. Table 1 summarises patient demographics including baseline pathology (neoplasia status) at the time of imaging, treatment history and BE length. A mean of $48 \pm 27 \mathrm{~mL}$ of water was consumed during the tethered capsule procedure. In all but one patient, tethered capsule imaging was followed by an oesophagogastroduodenoscopy (EGD). This patient was scheduled for an unrelated colonoscopy, but was in ongoing surveillance for long segment BE, with a recent EGD in the previous year. Therefore, this patient was not asked to provide toleration scores for endoscopy.

\section{Tissue contact with capsule and longitudinal coverage}

A mean of $5.5 \pm 1.3$ pullback datasets were obtained per patient, from which an optimal dataset was selected. In 16 patients, there was a $75 \% \pm 27 \%$ mean per cent of tissuecapsule contact averaged over the en face BE region of analysis. Longitudinal image coverage was $59 \% \pm 34 \%$ of the BE segment. Tissue contact was associated with endoscopic BE length and sliding hiatal hernia (figure 2). The mean tissue contact was $89 \% \pm 11 \%$ for short segment $\mathrm{BE}$ with/without prior ablative treatment $(\mathrm{n}=8)$ patients, and $61 \% \pm 31 \%$ for long segment $\mathrm{BE}(\mathrm{n}=8)(\mathrm{p}=0.03)$. The mean tissue contact was $84 \% \pm 15 \%$ in patients without sliding hiatal hernia $(n=11)$, and $55 \% \pm 37 \%$ with hernia $(\mathrm{n}=5) \quad(\mathrm{p}=0.04)$. Folds in the oesophagus sometimes occurred, suggesting that a larger percentage of the oesophagus was not imaged than suggested by the per cent of capsule circumference out of contact. Also, BE is not fully circumferential in patients with Prague $\mathrm{M}>\mathrm{C}$. Therefore, the percentage of $\mathrm{BE}$ imaged may be greater or less than that inferred from capsule contact.

\section{Toleration scores}

The mean procedure time for tethered capsule imaging was $9.7 \pm 3.0 \mathrm{~min}$. For capsule imaging $(\mathrm{n}=16)$, the mean preprocedure anxiety was $1.9 \pm 1.0$, procedural discomfort was $2.5 \pm 1.1$ and recommendation score was $1.3 \pm 0$.7. For 
Table 1 Patient demographics and clinical characteristics $(n=16)$

\begin{tabular}{|c|c|}
\hline Age, mean $( \pm S D)$ & $68(7)$ \\
\hline Sex, male, no. (\%) & $16(100)$ \\
\hline Race, white, no. (\%) & $16(100)$ \\
\hline \multicolumn{2}{|l|}{ Baseline pathology and treatment status } \\
\hline NDBE subjects, no. (\%) & $9(56)$ \\
\hline Short segment $(\leq 3 \mathrm{~cm}) \mathrm{BE}$, no. $(\%)$ & $2(13)$ \\
\hline LGD subjects, no. (\%) & $4(25)$ \\
\hline Ablative treatment-naïve subjects, no. (\%) & $1(6)$ \\
\hline Short segment BE, no. (\%) & $1(6)$ \\
\hline Treated subjects, no. (\%) & $3(19)$ \\
\hline Residual short segment BE, no. (\%) & $3(19)^{*}$ \\
\hline HGD/IMC subjects, no. (\%) & $3(19)$ \\
\hline Ablative treatment-naïve subjects, no. (\%) & $2(13) \dagger$ \\
\hline Short segment BE, no. (\%) & $1(6)$ \\
\hline Treated subjects, no. (\%) & $1(6)$ \\
\hline Residual short segment BE, no. (\%) & $1(6)$ \\
\hline \multicolumn{2}{|l|}{ Length of BE at study endoscopy, cm } \\
\hline Circumferential extent, mean $( \pm S D)$ & $3.6(4.3)$ \\
\hline Maximal extent, mean $( \pm S D)$ & $5.1(4.5)$ \\
\hline Short segment $(\leq 3 \mathrm{~cm})$ subjects, no. $(\%)$ & $8(50)$ \\
\hline Long segment $(>3 \mathrm{~cm}$ ) subjects, no. (\%) & $8(50)$ \\
\hline $\begin{array}{l}\text { Distance from diaphragmatic hiatus }(D) \text { to } \\
\text { gastric folds }(G) \text {, mean }( \pm S D)\end{array}$ & $2.3(2.5)$ \\
\hline $\begin{array}{l}\text { Subjects with sliding hiatal hernia (D-G >2 } \\
\mathrm{cm}) \text {, no. }(\%)\end{array}$ & $5(31)$ \\
\hline Length of hiatal hernia, mean $( \pm S D)$ & $5.6(2.1)$ \\
\hline
\end{tabular}

${ }^{*}$ One treated LGD patient had no visible BE on endoscopy and was classified as short segment BE.

†One HGD/IMC patient had prior endoscopic mucosal resection and no ablation, thus classified as ablative treatment-naïve. BE, Barrett's oesophagus; HGD, high-grade dysplasia; IMC, intramucosal carcinoma; LGD, low-grade dysplasia; NDBE, nondysplastic BE.

endoscopy $(\mathrm{n}=15)$, the mean preprocedure anxiety was $1.3 \pm 0.7$, procedural discomfort was $1.6 \pm 0.9$ and recommendation score was $1.1 \pm 0.3$. Toleration did not appear to be associated with the number of imaging pullbacks performed. The toleration score distributions, grouped by patient history of pathology/treatment are presented in figure 3.

Toleration scores stratified by pathology and treatment history were generally consistent between subgroups, suggesting that toleration may be independent of prior endoscopy and treatment experience. Endoscopy was better tolerated than the tethered capsule, likely due to sedation. Our toleration scores show marginally lower preprocedure anxiety $(1.9 \pm 1.1$ vs $2.1 \pm 0.8)$ and higher procedural discomfort $(2.5 \pm 1.0$ vs $1.9 \pm 0.9$ ) than previous reports, ${ }^{17}$ possibly due to our larger capsule diameter (12 vs $11 \mathrm{~mm}$ ) chosen for
Tissue contact over BE segment
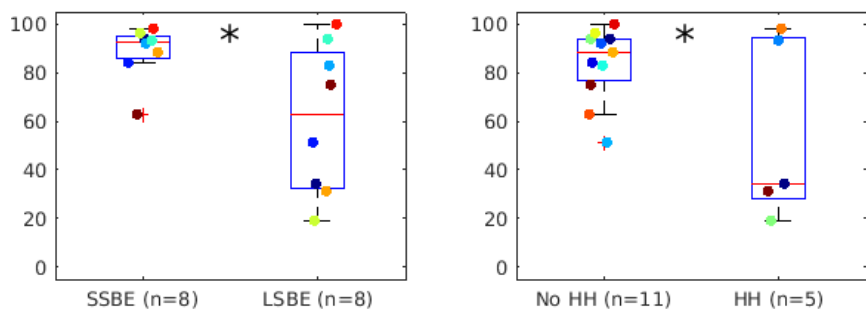

Longitudinal image coverage over BE segment
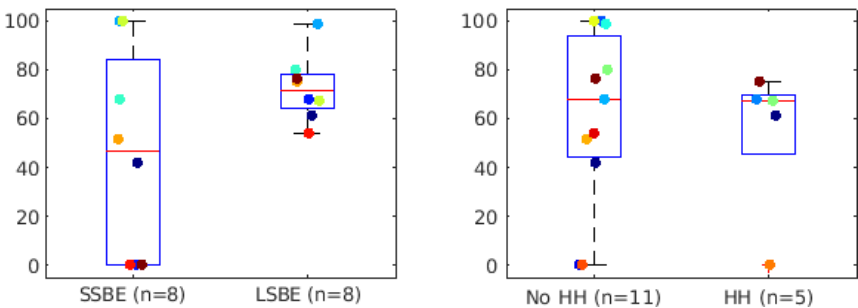

Figure 2 Box plots of tissue contact and longitudinal capsule motion uniformity/coverage over the en face Barrett's oesophagus $(\mathrm{BE})$ region in the tethered capsule optical coherence tomography (OCT) datasets. Coloured circles indicate individual data points. Tissue contact was significantly different $\left(^{*}\right)$ between short/long segment BE (SSBE, LSBE) $(p=0.03)$ and absence/presence of sliding hiatal hernia $(\mathrm{HH})(\mathrm{p}=0.04)$.

better tissue contact. These results suggest that tethered capsule OCT is well-tolerated, although both our study and the previous study were single centre and our study enrolment was all-male veterans, so results are not generalisable. There were no adverse events during the entire study.

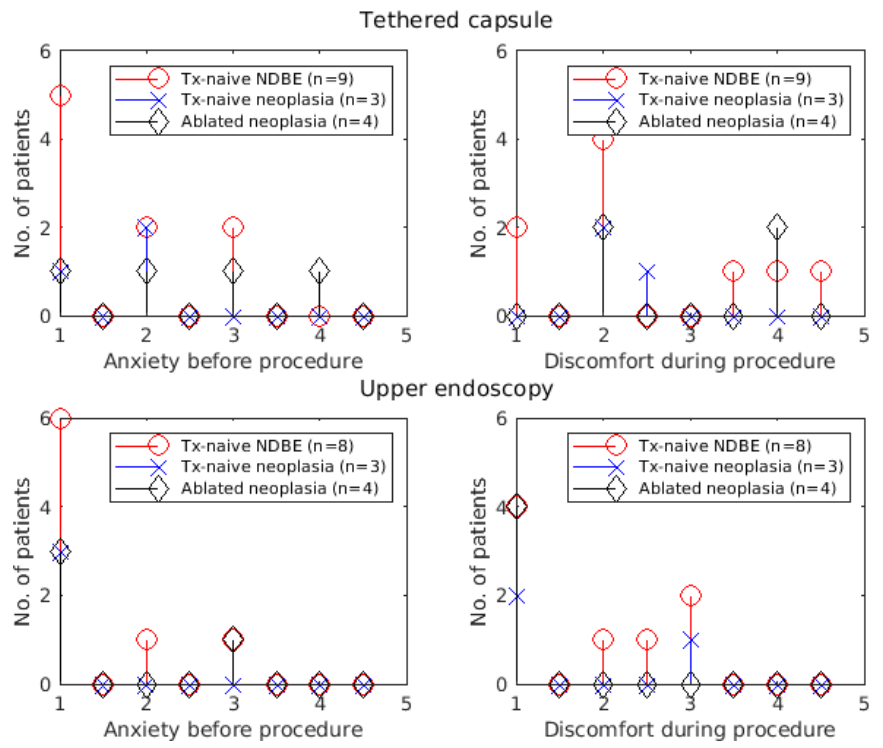

Figure 3 Preprocedure anxiety and procedural discomfort scores for the tethered capsule and endoscopy procedures. 1 , no anxiety/discomfort, 5, high anxiety/discomfort. Scores between patient subgroups of baseline pathology and treatment history were similar. NDBE, non-dysplastic Barrett's oesophagus. 


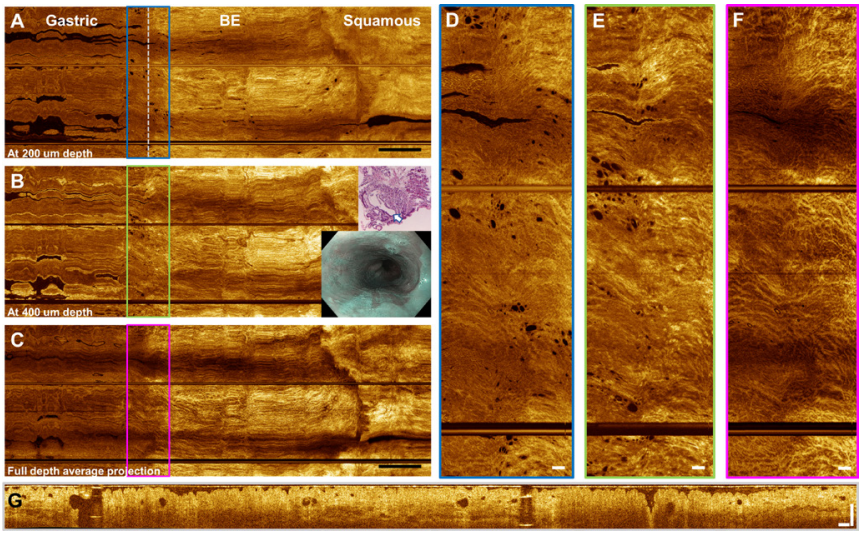

Figure 4 Tethered capsule optical coherence tomography (OCT) from a patient with C2M4 non-dysplastic Barrett's oesophagus (BE). Inset shows narrow band imaging view from a previous endoscopy. (A) En face OCT at $200 \mu \mathrm{m}$ depth, (B) $400 \mu \mathrm{m}$ depth and (C) full depth projection. Scale bars $1 \mathrm{~cm}$. Some longitudinal pullback non-uniformity can be observed in the BE segment. (D-F) Enlargements showing glands and mucosal pattern at the gastrooesophageal junction (GEJ). Scale bars $1 \mathrm{~mm}$. (G) Crosssectional OCT from the GEJ showing atypical glands. Scale bar $500 \mu \mathrm{m}$. Biopsy at the GEJ (inset) from a prior oesophagogastroduodenoscopy (9 months earlier) shows a large dilated cardiac gland (arrow) with smaller peripheral glands from the superficial mucosa.

\section{OCT features of BE}

Figure 4 shows a wide-field en face OCT image over an entire BE segment from the GEJ to SCJ. These data are from the patient who did not receive same-day EGD but had known long segment NDBE from two EGDs 9 months prior and 4 years prior (4-quadrant biopsies found no dysplasia). OCT showed almost complete contact of the oesophageal mucosa to the capsule, while some loss of longitudinal image coverage from longitudinal pullback non-uniformity was observed.

Numerous dilated glands were observed over the entire BE segment, and an exceptionally high number of gland clusters was observed on the gastric side of the GEJ. This example showed exceptionally well-defined boundaries of the GEJ and SCJ. This example also illustrates that important OCT markers may frequently occur at the gastric cardia around the GEJ, even in low-risk patients with NDBE. ${ }^{24}$ Features at the GEJ can confound attempts to identify neoplasia, particularly if the GEJ boundary is poorly defined and wide-field en face OCT views are unavailable. We report candidate OCT features for dysplasia in the online supplementary information. This information may be useful for future study design. In addition, findings suggestive of dysplasia on capsule OCT may be useful for informing follow-up endoscopy/ biopsy in the future.

\section{DISCUSSION}

We report a pilot study using ultrahigh-speed, tethered capsule SS-OCT imaging for wide-field, en face and cross-sectional visualisation. Previous studies with commercial OCT instruments used slower, 50000 A-scans/s, imaging speeds and lower, $40 \mu \mathrm{m}$ transverse resolution, generating sparsely spaced, cross-sectional image volumes that did not have high resolution en face views. Our OCT technology at 1000000 A-scans/s and $30 \mu \mathrm{m}$ resolution can generate densely sampled volumes enabling en face imaging over wide fields of view (online supplementary video 1). Previous studies used micromotor probes introduced into the endoscope working channel to generate en face and cross-sectional views enabling volumetric assessment of $\mathrm{BE}$ and dysplasia, but were limited to small fields of view. ${ }^{19}{ }^{20}$ We previously reported the feasibility of ultrahigh-speed tethered capsule OCT for volumetric and en face imaging $>20 \mathrm{~cm}$ lengths of the oesophagus in sedated patients during endoscopy, demonstrating large fields of view, detecting $\mathrm{BE}$ and suggesting features associated with dysplasia. ${ }^{21}$

Previous OCT capsule studies investigating screening recruited largely from a primary care population, whose patients had little or no BE, and those study designs did not use same-day endoscopy or biopsy histology for direct confirmation of BE. In this study, we recruited from a heterogeneous BE surveillance population in which patients had various BE lengths and history of dysplasia. Improved capsule design using lubricious materials and tapered form factor, as well as improved procedural workflow, increased capsule imaging performance. This study assessed procedures and performance for imaging unsedated patients before endoscopy; however, tethered capsule imaging can also be performed during endoscopy. This study did not attempt to assess the sensitivity/ specificity of the procedure. Sensitivity/specificity of OCT for detecting BE has been reported in prior studies. ${ }^{10}$ Future studies with a larger patient enrolment could be performed to prospectively assess sensitivity/specificity of OCT capsule imaging.

Limitations of this study include the lack of histological correlation with OCT features. Four-quadrant biopsies were performed in patients with NDBE history, but patients with prior treatment-naïve dysplasia referred for treatment were not systematically biopsied. We performed additional analyses of OCT features that may be associated with dysplasia (online supplementary information), with the caveat that these observations had histological corroboration on a per-patient basis, but not at the precise location where the OCT features were found. Our capsule could not mark areas of interest for biopsy or obtain biopsy directly. The tethered capsule procedure in its present form is not intended for confirmatory diagnosis of BE; it is intended as a screening procedure, enabling access to a larger population. Suspected BE on capsule imaging would be used to refer a patient for follow-up endoscopy and endoscopic surveillance if necessary. Capsule imaging might also be used to indicate the approximate location of possible dysplastic features by noting the longitudinal position of the lesion (via markings on the tether) and its 'clock position' (location 
around the circumference) from the OCT image map to inform follow-up endoscopy and biopsy. Laser marking based on cross-sectional image guidance using tethered capsules has been reported ${ }^{25}$; future capsule studies could adapt the laser marking paradigm to guide biopsy based on both en face and cross-sectional features.

Another limitation was the small patient enrolment from a single centre, therefore results on toleration, image coverage and image quality may not generalise to larger studies. However, this type of pilot study is important for optimising device design and examination protocols; identifying candidate OCT markers of $\mathrm{BE}$, dysplasia and risk stratification and designing future large prospective studies.

Close contact of the capsule with the oesophagus is critical for OCT visualisation, assuring that the circumference of the oesophagus is imaged and within the optical focus depth of field. Reduced oesophageal contact can introduce optical aberrations that reduce OCT visibility of mucosal microstructure, and oesophageal folds can obscure areas from view. These effects reduce image coverage and produce sampling errors. Previous studies suggested that peristaltic contractions initiated by swallowing would ensure contact between capsule and oesophagus. However, in our experience, patient swallows were helpful, but a substantial minority of patients had poor contact despite repeated dry/wet swallows to avoid deglutitive inhibition of the LES. The patients with short segment BE $(n=8)$ showed superior tissue contact compared with patients with long segment BE $(n=8)$ (contact $89 \pm 11 \%$ vs $61 \pm 31 \%$, $\mathrm{p}=0.03$ ) (figure 3 ). Patients without sliding hiatal hernia $(\mathrm{n}=11)$ showed superior contact compared with those with hernia $(n=5)$ (contact $84 \pm 15 \%$ vs $55 \pm 37 \%$, p=0.04). Early studies of oesophageal motility in $\mathrm{BE}$ found associations between long $\mathrm{BE}$ lengths and reduction of LES tone and peristaltic amplitude. ${ }^{26}$ The reduced LES tone associated with sliding hiatal hernia ${ }^{22}$ may also contribute to poor contact. In the future, contact might be improved using an articulating mechanism to appose the capsule to the oesophageal wall.

Longitudinal capsule motion uniformity is necessary for high quality en face visualisation. The capsule moved slower/faster than the tether pullback during portions of the OCT acquisitions, resulting in a localised stretched/ compressed appearance in en face images and sampling errors. Previous studies with micromotor imaging probes $^{27}$ used $2 \mathrm{~mm} / \mathrm{s}$ motorised pullback of the scanning optics within a transparent sheath to acquire volumetric OCT. Our tethered capsule was manually pulled back at $\sim 1 \mathrm{~cm} / \mathrm{s}$ while in contact with the oesophagus. In our previous study using a tethered capsule during sedated endoscopy, ${ }^{21}$ capsule motion was non-uniform at slow speeds due to friction. The lubricious housing used in the current capsule reduced friction and the faster 1 $\mathrm{cm} / \mathrm{s}$ pullback improved capsule longitudinal motion uniformity.
During pullback, the operator reported occasional resistance from anatomic variations, peristalsis, LES tone and/or rebound contractions, leading to longitudinal pullback non-uniformity. The operator did not forcefully pull through these resistances, which are meaningful anatomical signals for safe and effective use. Short segment BE had more non-uniform capsule motion compared with long segment BE, possibly because the contact produced by strong LES tone increased friction. Conversely, long segment BE had looser tissue-capsule contact, which may enable smoother capsule motion. Smooth pullback and capsule-tissue contact generally showed opposite (inverse) trends, because contact produced friction. Longitudinal pullback non-uniformity produces stretching/compression artefacts in the en face image; however, distortion occurs only in the pullback direction and image features can still be interpreted by experienced readers. Future attempts to perform more precise quantitative measurements or reduce sampling error in en face OCT images should incorporate more sophisticated motion artefact correction or motion tracking. Further increases in imaging speed will be possible and will enable faster pullbacks with reduced friction and improved longitudinal image coverage and uniformity, although pullback speeds may need to be moderated while traversing constrained sphincters.

Limitations in capsule contact and motion uniformity produce sampling error and suggest that the current version of the capsule would not be suitable for surveillance to detect focal pathologies such as dysplasia. However, capsules can be used for BE screening because BE occurs over large regions and sampling errors would not appreciably compromise detection sensitivity. In our study, tethered capsule OCT showed BE features in all patients having histologically confirmed BE.

For surveillance applications, a capsule imaging device might be used during sedation endoscopy. The capsule could be attached to the endoscope (similar to a focal radiofrequency ablation catheter) and articulated to contact the oesophageal wall, mapping the longitudinal extent of BE by advancing or retracting the endoscope. This protocol should enable comprehensive imaging coverage as well as access to the GEJ, areas of hiatal hernia and gastric cardia. The ultrahigh-speed tethered capsule produces better images than balloon OCT devices, should have better image coverage and could be multiuse, reducing cost.

Ultrahigh-speed tethered capsule OCT can visualise mucosal architectural morphology over wide fields of view. These technologies and methods may help the early screening of BE and risk stratification. Additional larger studies with histological correlation are warranted.

Contributors KL, JGF and HM designed the study. KL, 00A and BP developed the OCT imaging technology. KL, OOA, AM, JZ, THN, MF and HM collected the data. $\mathrm{KL}, 00 \mathrm{~A}$, JGF and HM analysed the data. QH made the histological diagnoses. JGF and HM obtained funding for the study. KL, JGF and HM wrote the manuscript. All authors read and contributed to the manuscript. JGF and HM were principal investigators for this study. 
Funding National Institutes of Health grants R01-CA075289-21 (JGF and HM) and R44CA235904- 02 (JGF), Veterans Administration Innovation Award (HM), graduate fellowship from Agency for Science, Technology and Research, Singapore (KL).

Competing interests None declared.

Patient consent for publication Not required.

Ethics approval The study was approved by IRBs at the Veterans Affairs Boston Healthcare System, Harvard Medical School and Massachusetts Institute of Technology.

Provenance and peer review Not commissioned; externally peer reviewed.

Data availability statement Data are available on reasonable request. Study data (OCT images, toleration scores) deidentified of patient information is available on reasonable request from the investigators and with approval from the Research Committees at the VA Boston Healthcare System, Harvard Medical School and MIT.

Open access This is an open access article distributed in accordance with the Creative Commons Attribution Non Commercial (CC BY-NC 4.0) license, which permits others to distribute, remix, adapt, build upon this work non-commercially, and license their derivative works on different terms, provided the original work is properly cited, appropriate credit is given, any changes made indicated, and the use is non-commercial. See: http://creativecommons.org/licenses/by-nc/4.0/.

ORCID iD

James G Fujimoto http://orcid.org/0000-0002-0828-4357

\section{REFERENCES}

1 Dulai GS, Guha S, Kahn KL, et al. Preoperative prevalence of Barrett's esophagus in esophageal adenocarcinoma: a systematic review. Gastroenterology 2002;122:26-33.

2 Chak A, Faulx A, Eng C, et al. Gastroesophageal reflux symptoms in patients with adenocarcinoma of the esophagus or cardia. Cancer 2006;107:2160-6

3 Ross-Innes CS, Debiram-Beecham I, O'Donovan M, et al. Evaluation of a minimally invasive cell sampling device coupled with assessment of trefoil factor 3 expression for diagnosing Barrett's esophagus: a multi-center case-control study. PLoS Med 2015;12:e1001780.

4 Ross-Innes CS, Chettouh $\mathrm{H}$, Achilleos A, et al. Risk stratification of Barrett's oesophagus using a non-endoscopic sampling method coupled with a biomarker panel: a cohort study. Lancet Gastroenterol Hepatol 2017;2:23-31.

5 Sami SS, Dunagan KT, Johnson ML, et al. A randomized comparative effectiveness trial of novel endoscopic techniques and approaches for Barrett's esophagus screening in the community. Am J Gastroenterol 2015;110:148-58.

6 Shariff MK, Varghese S, O'Donovan M, et al. Pilot randomized crossover study comparing the efficacy of transnasal disposable endosheath with standard endoscopy to detect Barrett's esophagus. Endoscopy 2016;48:110-6.

7 Chan DK, Zakko L, Visrodia KH, et al. Breath testing for Barrett's esophagus using exhaled volatile organic compound profiling with an electronic nose device. Gastroenterology 2017;152:24-6.

8 Moinova HR, LaFramboise T, Lutterbaugh JD, et al. Identifying DNA methylation biomarkers for non-endoscopic detection of Barrett's esophagus. Sci Trans/ Med 2018;10. doi:10.1126/scitransImed. aao5848. [Epub ahead of print: 17 Jan 2018].

9 Isenberg G, Sivak MV, Chak A, et al. Accuracy of endoscopic optical coherence tomography in the detection of dysplasia in Barrett's esophagus: a prospective, double-blinded study. Gastrointest Endosc 2005;62:825-31.

10 Rodriguez MAC, de Moura DTH, Ribeiro IB, et al. Volumetric laser endomicroscopy and optical coherence tomography in Barrett's esophagus: a systematic review and meta-analysis. Endosc Int Open 2019;7:E1078-91.

11 Wolfsen HC, Sharma P, Wallace MB, et al. Safety and feasibility of volumetric laser endomicroscopy in patients with Barrett's esophagus (with videos). Gastrointest Endosc 2015;82:631-40.

12 Leggett CL, Gorospe EC, Chan DK, et al. Comparative diagnostic performance of volumetric laser endomicroscopy and confocal laser endomicroscopy in the detection of dysplasia associated with Barrett's esophagus. Gastrointest Endosc 2016;83:880-8.

13 Swager A-F, Tearney GJ, Leggett CL, et al. Identification of volumetric laser endomicroscopy features predictive for early neoplasia in Barrett's esophagus using high-quality histological correlation. Gastrointest Endosc 2017:85:918-26.

14 Trindade AJ, Inamdar S, Smith MS, et al. Volumetric laser endomicroscopy in Barrett's esophagus: interobserver agreement for interpretation of Barrett's esophagus and associated neoplasia among high-frequency users. Gastrointest Endosc 2017;86:133-9.

15 Ramirez FC, Akins R, Shaukat M. Screening of Barrett's esophagus with string-capsule endoscopy: a prospective blinded study of 100 consecutive patients using histology as the criterion standard. Gastrointest Endosc 2008;68:25-31.

16 Gora MJ, Sauk JS, Carruth RW, et al. Imaging the upper gastrointestinal tract in unsedated patients using tethered capsule endomicroscopy. Gastroenterology 2013:145:723-5.

17 Gora MJ, Simmons LH, Quénéhervé L, et al. Tethered capsule endomicroscopy: from bench to bedside at a primary care practice. J Biomed Opt 2016;21:104001.

18 Gora MJ, Quénéhervé L, Carruth RW, et al. Tethered capsule endomicroscopy for microscopic imaging of the esophagus, stomach, and duodenum without sedation in humans (with video). Gastrointest Endosc 2018;88:830-40.

19 Tsai T-H, Ahsen OO, Lee H-C, et al. Endoscopic optical coherence angiography enables 3-dimensional visualization of subsurface microvasculature. Gastroenterology 2014;147:1219-21.

20 Ahsen OO, Liang K, Lee H-C, et al. Assessment of Barrett's esophagus and dysplasia with ultrahigh-speed volumetric en face and cross-sectional optical coherence tomography. Endoscopy 2019;51:355-9.

21 Liang K, Ahsen OO, Lee H-C, et al. Volumetric mapping of Barrett's esophagus and dysplasia with en face optical coherence tomography tethered capsule. Am J Gastroenterol 2016;111:1664-6.

22 Hershcovici T, Mashimo H, Fass R. The lower esophageal sphincter. Neurogastroenterol Motil 2011;23:819-30.

23 Standards of Practice Committee of the American Society for Gastrointestinal Endoscopy, Lichtenstein DR, Jagannath S, et al. Sedation and anesthesia in Gi endoscopy. Gastrointest Endosc 2008;68:815-26.

24 Trindade AJ, Raphael KL, Inamdar S, et al. Volumetric laser endomicroscopy features of dysplasia at the gastric cardia in Barrett's oesophagus: results from an observational cohort study. BMJ Open Gastroenterol 2019;6:e000340.

25 Gora MJ, Soomro AR, Puricelli WP, et al. 359 unsedated screening for Barrett's esophagus using tethered capsule endomicroscopy. Gastrointest Endosc 2014;79:AB136.

26 Loughney T, Maydonovitch CL, Wong RK. Esophageal manometry and ambulatory 24-hour pH monitoring in patients with short and long segment Barrett's esophagus. Am J Gastroenterol 1998;93:916.

27 Tsai T-H, Lee H-C, Ahsen OO, et al. Ultrahigh speed endoscopic optical coherence tomography for gastroenterology. Biomed Opt Express 2014;5:4387-404. 